\title{
Maternal and neonatal outcomes of pregnancy at 39 weeks and beyond with mild gestational diabetes mellitus
}

\author{
Hanqing Chen, Suhua Zou, Jianbo Yang, Songqing Deng, Jian Cai, Zilian Wang \\ First Affiliated Hospital of Sun Yat-sen University, China
}

\begin{abstract}
Objectives: The purpose of this study was to retrospectively analyze maternal and neonatal outcomes in pregnant women with mild gestational diabetes mellitus at 39 weeks compared to 40 weeks.

Material and methods: Clinical data of 372 cases of mild gestational diabetes mellitus form First Affiliated Hospital of Sun Yat-sen University were analyzed retrospectively. There were 108 mild GDM patients that delivered at $40-40^{+6}$ weeks in our research group, and 264 patients that delivered in $39-39^{+6}$ weeks in the control group. Neonatal and maternal outcomes were compared between the two groups.

Results: There was no difference between the two groups in the rate of cesarean section $(42.6 \%$ vs. $45.5 \%, p=0.614)$. The incidence of large for gestational age between the two groups was also not different ( $11.1 \%$ vs. $10.6 \%, p=0.887)$. The rate of postpartum hemorrhage and shoulder dystocia of the two groups was not different either $(p>0.05)$. There was no significant difference in the incidence of fetal distress, neonatal asphyxia, neonatal pathological jaundice, neonatal hypoglycemia, and neonatal respiratory distress syndrome in the two groups $(p>0.05)$.

Conclusions: There were no significant differences in adverse pregnancy outcomes and neonatal outcomes in women with mild gestational diabetes between deliveries at 39 and 40 weeks.
\end{abstract}

Key words: gestational diabetes mellitus, cesarean section, large for gestational age

Ginekologia Polska 2017; 88, 7: 366-371

\section{INTRODUCTION}

Gestational diabetes mellitus (GDM) is one of the most common complications in pregnancy. It is reported that the incidence of GDM varies from $1 \%$ to $20 \%$ [1-4]. Although the effects of GDM on mother and fetus have been widely recognized, there are still many problems to be solved. Previous studies have shown that elective induction at $38 \mathrm{com}-$ plete weeks reduces newborn weight and related complications [5]. American Diabetes Association (ADA) supported this in 2003 by pointing out that prolonging gestation past 38 weeks increased the risk of fetal macrosomia without reducing cesarean rates, so delivery during the 38th week shall be preferred [6]. In recent years however, it has been argued that the evidence of inducing deliveries at 38 weeks to prevent the occurrence of macrosomia and other GDM complications is insufficient $[7,8]$. Despite more and more understanding of GDM and the application of new standard GDM criteria released by International Association of Diabetes and Pregnancy Study Groups (IADPSG), the incidence of GDM has been rising significantly. Thus, the management of GDM patients may seem controversial. What is the appropriate timing to delivery then? It is widely agreed that a pregnant GDM woman should deliver before 40 weeks of gestation because of an increased risk of stillbirth. On the other hand however, based on conflicting findings in literature and our practice, such risk-increase seems not to apply in cases of mild GDM that is early well-controlled and uncomplicated. Therefore, it would be conclusive to find out the best delivery time in mild GDM cases. Therefore, the purpose of this study was to compare maternal and neonatal outcomes at 39 versus 40 weeks of mild GDM, and to provide additional clinical evidence of optimal delivery timing for mild GDM. 


\section{MATERIAL AND METHODS}

Data was collected from patients in obstetrics department of First Affiliated Hospital of Sun Yat-Sen University between March 2014 and August 2015. The data included: demographics, complications, delivery information as well as maternal and neonatal outcomes. The inclusion criteria included: (1) single pregnancy, vertex; (2) delivery by elective or emergency caesarean section and by spontaneous labor or induction at $\geq 39$ weeks; (3) failed oral glucose tolerance test (OGTT) during 24-28 weeks [9]; (4) satisfactory glycemia control; (5) no complications. Exclusion criteria included: multiple pregnancy; delivery at $<39$ weeks; pregnancy associated with other diseases or other obstetric complications such as intrahepatic cholestasis syndrome during pregnancy or severe infection; pre-gestational diabetes mellitus; poor glycemic control; GDM requiring medication for control. Patient information was collected from electronic documents and included age, body mass index (BMI), parity, weight gain, OGTT value, mode of delivery, postpartum hemorrhage and other complications, perineal laceration, shoulder dystocia, neonatal birth weight, fetal distress, neonatal asphyxia, respiratory distress syndrome.

Outcomes were categorized into maternal outcomes and neonatal outcomes. Maternal outcomes include pregnancy induced hypertension, postpartum hemorrhage, and perineal laceration. Neonatal outcomes include shoulder dystocia, macrosomia, large for gestational age (LGA), small for gestational age (SGA), birth injury, neonatal death, neonatal hypoglycemia, fetal distress, neonatal asphyxia.

In this study the criteria used to diagnose GDM were those of IADPSG [9]. The fasting blood glucose test (FBG) was performed at the very first prenatal examination to exclude pre-gestational diabetes (FBG $\geq 7.00 \mathrm{mmol} / \mathrm{L}$ ). All patients in this study underwent a 2-hour $75 \mathrm{~g}$ oral glucose tolerance test at 24-28 gestation weeks with readings at $0 \mathrm{~h}, 1 \mathrm{~h}$ and 2 hours. GDM was diagnosed when any one of the $75 \mathrm{~g}$ OGTT conditions was met: FBG $\geq 5.1[\mathrm{mmol} / \mathrm{L}] ; 1 \mathrm{~h}$ after load $\geq 10.0$ [mmol/L]; $2 \mathrm{~h}$ after load $\geq 8.5[\mathrm{mmol} / \mathrm{L}]$. All patients were taught to control diet, exercise and monitor blood glucose. Mild GDM patients, to control their blood glucose level, needed diet control and exercise therapy only. Satisfaction with blood glucose levels was measured in Patients with fasting blood glucose $<5.3$ [mmol/L] and postprandial glucose at 1 hour $<7.8[\mathrm{mmol} / \mathrm{L}]$ or at 2 hours $<6.7[\mathrm{mmol} / \mathrm{L}]$ after eating were considered to have satisfactory blood glucose levels and did not require medication. All clinical investigations were conducted in accordance with the guidelines of the Declaration of Helsinki and were approved by the Hospital Ethics Committee [No. 2014(93)]. Informed consent was obtained from all participating parties.

\section{Statistical methods}

Maternal and delivery data was described by percentages and mean \pm SD. Categorical variables were compared using Chi-square test or Fisher's exact test while continuous variables using unpaired Student's $t$ test. Statistical analysis was carried out using IBM SPSS statistical package version 19.0. The $p$ value $<0.05$ was considered as statistically significant.

\section{RESULTS}

During the studied period there were 5145 cases of deliveries in our hospital, of which 967 cases (18.8\%) were diagnosed as gestational diabetes mellitus. There was a total of 435 of GDM deliveries beyond 39 weeks but 63 cases were excluded from the study, of which 13 due to pre-gestational diabetes mellitus, 27 due to unsatisfactory blood glucose control, 16 due to incomplete records and 7 due to insufficient monitoring of blood glucose. Consequently, there were 372 cases that met the study criteria: 264 deliveries at $39-39^{+6}$ weeks and 108 deliveries beyond 40 complete gestation weeks. Maternal age ranged from 18 to 44 years, with an average of $31.6 \pm 3.9$ years.

The weight gain during pregnancy in the study group was significantly higher than that in the control group $(13.20 \pm 4.54[\mathrm{~kg}]$ vs. $11.53 \pm 3.72[\mathrm{~kg}], \mathrm{p}<0.001)$. In the study group, the proportion of multiparas was significantly lower than that of the control group ( $17.6 \%$ vs. $31.1 \%, p=0.008)$. There was no significant difference in mode of conception, spontaneous abortion rate, OGTT value at $0 \mathrm{~h}$ (FBG) or at $1 \mathrm{~h}$, nor in glycosylated hemoglobin level $\left(\mathrm{HbA}_{1 \mathrm{c}}\right)(\mathrm{p}>0.05)$ between the two groups. The data is summarized in Table 1 .

Also, the two groups showed no significant difference in cesarean section rate, postpartum hemorrhage, shoulder dystocia or cervical laceration ( $p>0.05$ ). We recorded a second degree perineal laceration in only one case and none of severe damage ( $3^{\text {rd }}$ or $4^{\text {th }}$ degree). The pregnancy outcomes and complications are presented in Table 2.

Similarly, there was no significant difference in other adverse events such as the rate of macrosomia, fetal distress, neonatal asphyxia, LGA, neonatal pathological jaundice, neonatal hypoglycemia or neonatal respiratory distress syndrome (RDS) $(p>0.05)$. There was no significant difference between the two groups in SGA either, but the proportion of SGA in the study group was lower than that in the control group (5.6\% vs. $11.7 \%, p=0.070$ ). There was no intrauterine fetal death recorded. The average neonatal weight of the study group was slightly higher than that of the control group $(3.33 \pm 0.35[\mathrm{~kg}]$ vs. $3.25 \pm 0.38[\mathrm{~kg}], p=0.042)$. The neonatal outcomes in the two groups are summarized in Table 3.

\section{DISCUSSION}

The diagnostic criteria for gestational diabetes mellitus vary across countries and regions. Consequently, the 
Table 1. Basic data of the two groups

\begin{tabular}{|c|c|c|c|c|}
\hline Items & Research group & Control group & $x^{2}$ & $P$ value \\
\hline N & 108 & 264 & & \\
\hline Age (years) & $30.60 \pm 3.49$ & $32.00 \pm 3.93$ & $3.224^{*}$ & 0.001 \\
\hline$\geq 35$ years & $17(15.7 \%)$ & $63(23.9 \%)$ & 2.996 & 0.083 \\
\hline Mean abortions & $0.51 \pm 0.80$ & $0.62 \pm 0.93$ & $1.060^{*}$ & 0.290 \\
\hline \multicolumn{5}{|l|}{ Conception } \\
\hline Natural & $99(91.7 \%)$ & $234(88.6 \%)$ & 0.750 & 0.386 \\
\hline Artificial & $9(8.3 \%)$ & $30(11.4 \%)$ & & \\
\hline \multicolumn{5}{|l|}{ BMI before delivery } \\
\hline $18.9-24.9\left[\mathrm{~kg} / \mathrm{m}^{2}\right]$ & $32(29.6 \%)$ & $104(39.4 \%)$ & & \\
\hline $25.0-29.9\left[\mathrm{~kg} / \mathrm{m}^{2}\right]$ & $56(51.9 \%)$ & $137(51.9 \%)$ & & \\
\hline$\geq 30\left[\mathrm{~kg} / \mathrm{m}^{2}\right]$ & $20(18.5 \%)$ & $23(8.7 \%)$ & 8.375 & 0.015 \\
\hline Weight gain [kg] & $13.20 \pm 4.54$ & $11.53 \pm 3.72$ & -3.668 & $<0.001$ \\
\hline Primipara & $89(82.4 \%)$ & $182(68.9 \%)$ & 7.029 & 0.008 \\
\hline Multipara & $19(17.6 \%)$ & $82(31.1 \%)$ & & \\
\hline \multicolumn{5}{|l|}{ Mean OGTT reading $[\mathrm{mmol} / \mathrm{L}]$} \\
\hline At $0 \mathrm{~h}$ (FBG) & $4.72 \pm 0.46$ & $4.74 \pm 0.57$ & 0.198 & 0.843 \\
\hline At $1 \mathrm{~h}$ & $9.72 \pm 1.35$ & $9.76 \pm 1.50$ & 0.248 & 0.804 \\
\hline At $2 \mathrm{~h}$ & $8.59 \pm 1.18$ & $8.95 \pm 1.25$ & 2.568 & 0.011 \\
\hline $\mathrm{HbA}_{1 \mathrm{c}}(\%)$ & $5.37 \pm 0.50$ & $5.41 \pm 0.38$ & 0.794 & 0.427 \\
\hline \multicolumn{5}{|c|}{ \# of cases with abnormal values at any of the 3 OGTT readings } \\
\hline Any 1 out of 3 & $65(60.2 \%)$ & $140(53.0 \%)$ & & \\
\hline Any 2 out of 3 & $36(33.3 \%)$ & $95(36.0 \%)$ & & \\
\hline All 3 readings abnormal & $7(6.5 \%)$ & $29(11.0 \%)$ & 2.471 & 0.291 \\
\hline
\end{tabular}

*Student's $t$ test; BMI — body mass index; OGTT — oral glucose tolerance test; $\mathrm{FPG}$ - fasting plasma glucose; $\mathrm{HbA}_{1 c}$ - hemoglobin $\mathrm{A}_{1 \mathrm{c}}$

Table 2. Outcomes and complications of the two groups

\begin{tabular}{|l|c|c|c|c|}
\hline Items & Research group & Control group & $\mathbf{X}^{2}$ & \\
\hline $\mathrm{N}$ & 108 & 264 & & \\
\hline Spontaneous labor & $53(49.1 \%)$ & $129(48.9 \%)$ & & 0.614 \\
\hline Forceps & $9(8.3 \%)$ & $15(5.7 \%)$ & 0.977 & 0.805 \\
\hline Cesarean section & $46(42.6 \%)$ & $120(45.5 \%)$ & & 0.871 \\
\hline Pregnancy hypertension & $9(8.3 \%)$ & $20(7.6 \%)$ & 0.061 & $1.000^{*}$ \\
\hline Postpartum hemorrhage & $15(13.9 \%)$ & $35(13.3 \%)$ & 0.026 & 0.346 \\
\hline Vaginal injury & $3(2.8 \%)$ & $7(2.7 \%)$ & - & $0.419^{*}$ \\
\hline Cervical laceration & $8(7.4 \%)$ & $13(4.9 \%)$ & 0.887 & - \\
\hline Shoulder dystocia & $3(2.8 \%)$ & $4(1.5 \%)$ & & \\
\hline
\end{tabular}

*Fisher's exact test

incidence of GDM is different and may vary even within the same country, as standards are not uniform. The incidence of GDM in China has reached $17.5 \%$ according to data from a multicenter clinical trial in China [10]. Although there is now a greater understanding of GDM and most cases are well managed having a satisfactory glycemic control, the
GDM complications and fetal macrosomia are still on a rise significantly [11]. Maternal high blood glucose crosses placenta and circulates in the fetus stimulating fetal insulin secretion. That leads to increased anabolism and deposition of fat and glycogen in the fetal tissue, hence fetal macrosomia. Such newborns have higher risk of shoulder dystocia, bra- 


\begin{tabular}{|c|c|c|c|c|}
\hline Items & Research group & Control group & $x^{2}$ & $P$ value \\
\hline $\mathrm{N}$ & 108 & 264 & & \\
\hline Weight [kg] & $3.33 \pm 0.35$ & $3.25 \pm 0.38$ & $-1.976^{*}$ & 0.049 \\
\hline Macrosomia & $4(3.7 \%)$ & $11(4.2 \%)$ & - & $1.000^{\#}$ \\
\hline Fetal distress & $35(32.4 \%)$ & $77(29.2 \%)$ & 0.383 & 0.536 \\
\hline Neonatal asphyxia & $1(0.9 \%)$ & $8(3.0 \%)$ & - & $0.457^{\#}$ \\
\hline Male & $56(51.9 \%)$ & $138(52.3 \%)$ & 0.005 & 0.941 \\
\hline LGA & $12(11.1 \%)$ & $28(10.6 \%)$ & 0.020 & 0.887 \\
\hline SGA & $6(5.6 \%)$ & $31(11.7 \%)$ & 3.275 & 0.070 \\
\hline Jaundice & $23(21.3 \%)$ & $40(15.2 \%)$ & 2.057 & 0.151 \\
\hline Hypoglycemia & $6(5.6 \%)$ & $21(8.0 \%)$ & 0.655 & 0.418 \\
\hline RDS & $2(1.9 \%)$ & $3(1.1 \%)$ & - & $0.630^{\#}$ \\
\hline
\end{tabular}

*Student's $t$ test, "Fisher's exact test; LGA — large for gestational age; SGA — small for gestational age; RDS — neonatal respiratory distress syndrome

chial plexus injury and neonatal asphyxia. Therefore, previous studies and guidelines have recommended the use of elective delivery method within 38 weeks to minimize macrosomia and associated complications. In 2008 National Institute for Health \& Clinical Excellence (NICE) in UK recommended that a GDM fetus, if normal, could be delivered by induction at 38 weeks of gestation or by elective cesarean section [12]. However, such strategy has been controversial while practice showed it was far from easy to determine the optimal time for delivery. It is generally agreed that a GDM pregnant women should deliver before 40 weeks. In recent years, the discussion about the appropriate timing of delivery of GDM has been focused on the necessity to intervene either before or after 40 weeks of gestation, where in case of the latter, the risk of fetal distress and fetal death may increase. Eman et al. thought that the recommendations on optimal time window for delivery in GDM are not precise enough and have weak foundations. Simply, there is no evidence that electively induced labor is any better than spontaneous natural labor past 40 weeks. The authors further suggested that in GDM cases with ideal blood glucose control and no complications it is not necessary at all to deliver at 40 weeks the latest [4]. Mpondo et al. [7] suggested that despite the common belief that GDM pregnancy should be delivered at term, there was no adequate evidence for ideal timing and mode of delivery in GDM cases based on retrospective analysis. Sutton et al. [13] have analyzed retrospectively a total of 679 cases with mild GDM. The results showed that GDM deliveries before 40 weeks did not increase the cesarean section rate. In contrast, the incidence of Cesarean section of GDM at 40 weeks increased, but only in the induced group when compared to expected pregnancy at 40 weeks. Cesarean section rate increased significantly delivering beyond 40 weeks. In their comparison however the authors did not take into consideration the pregnancy outcomes nor the neonatal outcomes. Witkop et al. [14] have reviewed a few studies and found that in case of GDM pregnancy with good glycemic control, the incidence of macrosomia and associated complications can be reduced if management is active (induction at term) rather than expectant. In 2015 NICE brought to the attention that there was no convincing evidence that GDM pregnancy with appropriately managed glycemia has still an increased risk of a stillbirth but there was sufficient evidence to recommend induction of labor prior to 40 weeks of gestation to reduce the risk of shoulder dystocia [15].

The elective induction of labor is considered to be routine management of pregnant women with GDM in order to reduce the incidence of macrosomia and related complications. However, selective induction of labor was associated with prolonged labor, fetal distress, increased cesarean section rate, and shoulder dystocia [16-19]. Witkop et al. have compared elective induction with expectant management. The results showed that elective induction can significantly reduce the rate of LGA and shoulder dystocia, but due to immature cervical ripening of early induction, the failure rate increased. The elective induction at less than 38 weeks has a higher rate of vaginal delivery failure, which results with transit to cesarean section anyways [14]. The results of our study show that there is no difference in the rate of cesarean section, the rate of difficult birth, the incidence of macrosomia or the incidence of neonatal hypoglycemia whether delivered before or after 40 weeks. Such findings may be due to well-implemented strict procedures at our hospital; all study participants had a very well controlled glycemia. Another reason may be on account of the use of the GDM criteria from IADPSG recently implemented at our center, which are more inclusive (not satisfying any 
of the 3 OGTT readings classifies as GDM). This resulted in some of our cases to be classified as GDM, although they were not GDM under criteria previously used by us. We had a total of 15 cases of macrosomia in our study. The rate of macrosomia was not significantly differences between the two groups. The incidence of macrosomia was low because of strict management in this study including health education, diet control, exercise and blood glucose control. The effect was that none of the study participants needed insulin or oral medication. Such education was possible thanks to GDM day clinics that in China play an important role in the management of GDM pregnancies. Interestingly, this study showed that the percentage of SGA in 40 weeks of pregnancy was lower than that in the control group (5.6\% vs. $11.7 \%$, $p=0.070$ ), which is in line with intuition due to extra week of growth. We hypothesized that a well-managed strict glycemic control was the major factor for the overall SGA rate while we recorded no case of maternal hypoglycemia. Also, it may be that the strict GDM criteria of IADPSG that classify a previously normal pregnancy as abnormal glycemia pregnancy cause inclusion to the study and the management of GDM. When it comes to the neonatal weight, although the average weight in the 40 weeks group was significantly higher than that in the 39 weeks group, the absolute difference was very small.

The difference between the present study and other studies was that patients who participated in this study were mild GDM cases where mild is defined as that with good glycemic control due to exercise and adequate diet only. This way we minimize the interference factor of different diabetes types. The mean weight gain during pregnancy of the study group was bigger than that of the control group $(13.20 \pm 4.54$ vs. $11.53 \pm 3.72[\mathrm{~kg}], \mathrm{p}<0.001)$.

There was no difference between the two groups in shoulder dystocia. Interestingly, all shoulder dystocias occurred in non-macrosomic cases. Consequently, fetal weight less than 4000 [g] does not completely exclude the risk of shoulder dystocia. Fetal fat distribution and delivery mechanisms may be important risk factors for shoulder dystocia.

There was no fetal death in this study, which may be related to the strict blood glucose control, monitoring and management during pregnancy. This suggests that a strict control of blood glucose is essential to improve neonatal outcome.

\section{CONCLUSIONS}

It has been shown that it is not necessary to choose cesarean section to prevent macrosomia and related complications for patients with mild GDM. The results of this study showed that mild GDM pregnancies delivered beyond 39 weeks did not increase the adverse maternal and neonatal outcomes compared to pregnancy resolution at 39 weeks. Since this study is a retrospective study, it has some inherent limits. More randomized controlled studies are needed to examine the risks and benefits of a delayed delivery.

\section{Acknowledgements}

Hanqing Chen has contributed to the concept of this paper, to the analysis and interpretation of data and he has composed the article draft. Suhua Zou has helped to perform the analysis of data. Jianbo Yang has performed the data analyses and has written the manuscript. Songqing Deng has contributed to both the analysis and interpretation of data. Jian Cai has assisted in the writing of the manuscript. Zilian Wang had raised the idea of this article and contributed to the study design and manuscript revision.

\section{REFERENCES}

1. Farrar D, Duley L, Medley N, et al. Different strategies for diagnosing gestational diabetes to improve maternal and infant health. Cochrane Database Syst Rev. 2015; 1: CD007122, doi: 10.1002/14651858. CD007122.pub3, indexed in Pubmed: 25604891.

2. Tieu J, McPhee AJ, Crowther CA, et al. Screening and subsequent management for gestational diabetes for improving maternal and infant health. Cochrane Database Syst Rev. 2014(2): CD007222, doi: 10.1002/14651858.CD007222.pub3, indexed in Pubmed: 24515533.

3. Kelley KW, Carroll DG, Meyer A. A review of current treatment strategies for gestational diabetes mellitus. Drugs Context. 2015; 4: 212282, doi: 10.7573/dic.212282, indexed in Pubmed: 26213555.

4. Alfadhli EM. Gestational diabetes mellitus. Saudi Med J. 2015; 36(4): 399-406, doi: 10.15537/smj.2015.4.10307, indexed in Pubmed: 25828275.

5. Alberico S, Businelli C, Wiesenfeld U, et al. Gestational diabetes and fetal growth acceleration: induction of labour versus expectant management. Minerva Ginecol. 2010; 62(6): 533-539, indexed in Pubmed: 21079575.

6. American Diabetes Association. Gestational diabetes mellitus. Diabetes Care. 2003; 26(Suppl 1): S103-S105.

7. Mpondo BCT, Ernest A, Dee HE. Gestational diabetes mellitus: challenges in diagnosis and management. J Diabetes Metab Disord. 2015; 14: 42, doi: 10.1186/s40200-015-0169-7, indexed in Pubmed: 25977899.

8. Nicholson WK, Wilson LM, Witkop CT, et al. Therapeutic management, delivery, and postpartum risk assessment and screening in gestational diabetes. Evid Rep Technol Assess (Full Rep). 2008(162): 1-96, indexed in Pubmed: 18457474.

9. Metzger BE, Gabbe SG, Persson B, et al. International Association of Diabetes and Pregnancy Study Groups Consensus Panel. International association of diabetes and pregnancy study groups recommendations on the diagnosis and classification of hyperglycemia in pregnancy. Diabetes Care. 2010; 33(3): 676-682, doi: 10.2337/dc09-1848, indexed in Pubmed: 20190296.

10. Zhu WW, Yang HX, Wei YM, et al. Evaluation of the value of fasting plasma glucose in the first prenatal visit to diagnose gestational diabetes mellitus in China. Diabetes Care. 2013;36(3):586-590, doi: 10.2337/dc121157, indexed in Pubmed: 23193214.

11. Fadl HE, Ostlund IKM, Magnuson AFK, et al. Maternal and neonatal outcomes and time trends of gestational diabetes mellitus in Sweden from 1991 to 2003. Diabet Med. 2010; 27(4): 436-441, doi: 10.1111/j.1464-5491.2010.02978.x, indexed in Pubmed: 20536516.

12. Guideline Development Group. Management of diabetes from preconception to the postnatal period: summary of NICE guidance. BMJ. 2008; 336(7646): 714-717, doi: 10.1136/bmj.39505.641273.AD, indexed in Pubmed: 18369227.

13. Sutton AL, Mele L, Landon MB, et al. Eunice Kennedy Shriver National Institute of Child Health and Human Development Maternal-Fetal Medicine Units Network. Delivery timing and cesarean delivery risk in women with mild gestational diabetes mellitus. Am J Obstet Gynecol. 2014; 211(3): 244.e1-244.e7, doi: 10.1016/j.ajog.2014.03.005, indexed in Pubmed: 24607755.

14. Witkop CT, Neale D, Wilson LM, et al. Active compared with expectant delivery management in women with gestational diabetes: a systematic review. Obstet Gynecol. 2009; 113(1): 206-217, doi: 10.1097/ /AOG.0b013e31818db36f, indexed in Pubmed: 19104376. 
15. National Institute for Health and Care Excellence. Diabetes in pregnancy: management of diabetes and its complications from preconception to the postnatal period.NG3. 2015. www.nice.org.uk/guidance/ng3.

16. Lee HR, Kim MN, You JiY, et al. Risk of cesarean section after induced versus spontaneous labor at term gestation. Obstet Gynecol Sci. 2015; 58(5): 346-352, doi: 10.5468/ogs.2015.58.5.346, indexed in Pubmed: 26430658.

17. Bas-Lando $M$, Srebnik $N$, Farkash $R$, et al. Elective induction of labor in women with gestational diabetes mellitus: an intervention that modifies the risk of cesarean section. Arch Gynecol Obstet. 2014; 290(5): 905-912, doi: 10.1007/s00404-014-3313-6, indexed in Pubmed: 24973018.

18. Øverland EA, Vatten $L$, Eskild A. Pregnancy week at delivery and the risk of shoulder dystocia: a population study of 2,014,956 deliveries. BJOG. 2014; 121(1):34-41, doi: 10.1111/1471-0528.12427, indexed in Pubmed:24020942.

19. Glantz JC. Term labor induction compared with expectant management. Obstet Gynecol. 2010; 115(1): 70-76, doi: 10.1097/AOG.0b013e3181c4ef96, indexed in Pubmed: 20027037. 